\title{
Case Report \\ Impact of Heart Transplantation on Cheyne-Stokes Respiration in a Child
}

\author{
Suhail Al-Saleh, ${ }^{1,2}$ Paul F. Kantor, ${ }^{3}$ and Indra Narang ${ }^{1,2}$ \\ ${ }^{1}$ Division of Respiratory Medicine, Hospital for Sick Children, 555 University Avenue, Toronto, ON, Canada M5G 1X8 \\ ${ }^{2}$ University of Toronto, 27 King's College Cir, Toronto, ON, Canada M5S 2J7 \\ ${ }^{3}$ Division of Cardiology, University of Alberta, Stollery Children's Hospital, 8440112 Street Northwest, Edmonton, \\ $A B$, Canada T6G $2 B 7$ \\ Correspondence should be addressed to Suhail Al-Saleh; suhail.al-saleh@sickkids.ca
}

Received 6 February 2016; Accepted 27 March 2016

Academic Editor: Albert M. Li

Copyright (C) 2016 Suhail Al-Saleh et al. This is an open access article distributed under the Creative Commons Attribution License, which permits unrestricted use, distribution, and reproduction in any medium, provided the original work is properly cited.

Sleep disordered breathing is well described in adults with heart failure but not in pediatric population. We describe a 13-yearold Caucasian male with severe heart failure related to dilated cardiomyopathy who demonstrated polysomnographic features of Cheyne-Stokes respiration, which completely resolved following cardiac transplantation. Cheyne-Stokes respiration in children with advanced heart failure and its resolution after heart transplant can be observed similar to adults.

\section{Introduction}

In adults with heart failure (HF), Cheyne-Stokes respiration (CSR) is found to be a common polysomnographic (PSG) finding [1]. CSR is a type of central sleep apnea (CSA) that is characterized by consecutive episodes of central apneas and/or hypopneas separated by crescendo and decrescendo breathing patterns [2]. Although CSR is secondary to HF, its presence has negative physiologic effects on cardiovascular system and it is an independent risk factor for mortality in adults with $\mathrm{HF}[1,3,4]$. In children only two case reports have described CSR $[5,6]$. We describe in this report an adolescent with dilated cardiomyopathy (DCM) who had evidence of CSR on polysomnography (PSG) which was normalized following heart transplantation.

\section{Case Presentation}

The patient, a 13-year-old Caucasian male, presented with DCM and, after progressive deterioration of his ejection fraction, eventually required admission to the hospital for progressive HF. His symptoms were increasing daytime tiredness and lethargy as well as history of syncopal episodes and increased dyspnea on effort which all were attributed to the severe heart failure. His symptoms were New York Heart Association (NYHA) class III. Specifically, echocardiography showed deterioration of his cardiac function (decreased left ventricular ejection fraction [LVEF] from $30 \%$ to $9 \%$ over a two-month period). Medical therapy had included Digoxin, Carvedilol, Ramipril, and Spironolactone. He underwent a standard PSG according to international guidelines at the time of admission that was part of a prospective observational study to describe sleep disordered breathing (SDB) in children with known cardiomyopathy, the group data of which have been published elsewhere [7]. Significant findings in the PSG were a significantly elevated central apnea-hypopnea index (CAHI) of 17 events/hour with CSR similar to that described in adults with CSR (Figure 1). He remained in the hospital for pretransplantation assessment. During this time, he was transferred to the cardiac intensive care unit and started on intravenous Milrinone.

Four weeks after the baseline PSG, and before any treatment was initiated specifically for CSR, the patient underwent an orthotropic heart transplant. Following this, he was able to recover to normal NYHA status, with no symptoms, and normal cardiac function (LVEF was 61\%). A follow-up 


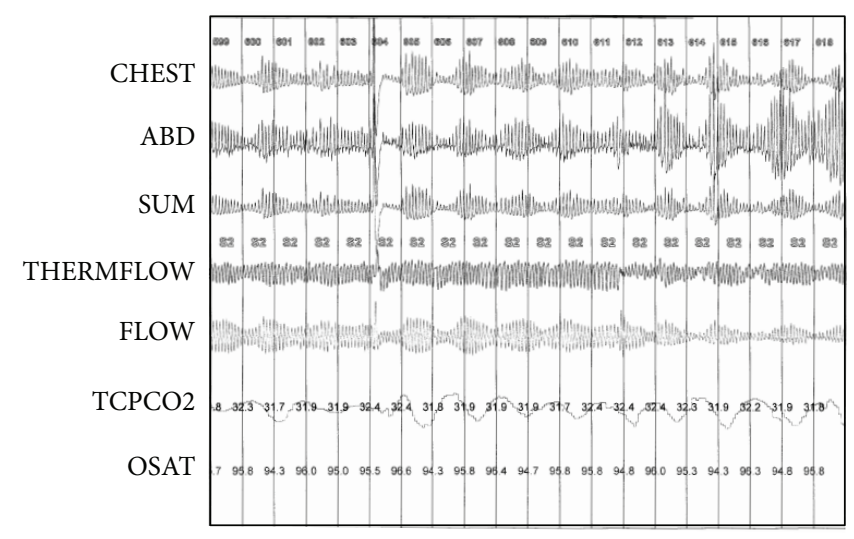

FIGURE 1: Cheyne-Stokes respiration in the child before heart transplant.

TABLE 1: PSG results of the patient before and after heart transplant.

\begin{tabular}{|c|c|c|}
\hline Results & Pretransplant & Posttransplant \\
\hline TST, min & 358.5 & 389.5 \\
\hline Sleep latency, min & 10.8 & 1.8 \\
\hline Sleep efficiency, \% & 96.5 & 91.5 \\
\hline REM latency, min & 159 & 167 \\
\hline Stage $1 \%$ TST & 0.6 & 1.4 \\
\hline Stage $2 \%$ TST & 59.6 & 54 \\
\hline Slow wave sleep \% TST & 21.2 & 24.9 \\
\hline REM \% TST & 18.7 & 19.6 \\
\hline Arousals, total index & 32.8 & 10 \\
\hline Mean sleep $\mathrm{SaO}_{2}(\%)$ & 93 & 98 \\
\hline Minimum $\mathrm{SaO}_{2}(\%)$ & 85 & 94 \\
\hline Highest $\mathrm{TcCO}_{2} /$ etCO $(\mathrm{mmHg})$ & 35 & 45 \\
\hline OAHI (events/hour) & 1.2 & 0.5 \\
\hline CAHI (events/hour) & 17.6 & 0.9 \\
\hline LVEF\% (Simpsons) & 9 & 61 \\
\hline
\end{tabular}

CAHI: central apnea-hypopnea index, etCO ${ }_{2}$ : end tidal carbon dioxide, LVEDd: left ventricular diastolic dimension, LVEF: left ventricular ejection fraction, OAHI: obstructive apnea-hypopnea index, REM: rapid eye movements, $\mathrm{SaO}_{2}$ : oxygen saturation, $\mathrm{TcCO}_{2}$ : transcutaneous carbon dioxide, and TST: total sleep time.

baseline PSG was done 3 months after heart transplantation to reevaluate his sleep related breathing. At this time point, he was clinically stable. His medications at this time included Prednisone, Mycophenolate Mofetil, and Tacrolimus. His repeat PSG showed that his breathing pattern had normalized with no further evidence of CSR. Table 1 describes Echocardiographic and PSG characteristics both before and after heart transplant.

\section{Discussion}

In adults, CSR has been described in detail in patients with HF [1], with reported prevalence of CSR in patients with advanced $\mathrm{HF}$ of up to $37 \%$ [8]. Importantly, its presence can be a predictor of mortality $[3,4]$. To our knowledge, this is the first comprehensive case report describing the presence of CSR in a child with cardiomyopathy and severely impaired cardiac function that completely resolved following heart transplantation.

Central apneas during sleep occur when $\mathrm{PaCO}_{2}$ falls below the apnea threshold. CSR is present when central apneas occur in cyclical fashion. The phenomenon is thought to be related to respiratory control system instability as well as a delayed circulatory time in association with low cardiac output in HF [1]. This occurs because of pulmonary vagal irritant receptor stimulation by pulmonary congestion in heart failure and increases in central and peripheral chemosensitivity [1]. The pathophysiology of respiratory control system instability leading to CSR is thought to be that of chronic hyperventilation leading to a decrease in $\mathrm{PaCO}_{2}$ which drops to a level close to the apnea threshold. Since patients with $\mathrm{HF}$ and CSR have lower $\mathrm{PaCO}_{2}$ than those without CSR during sleep [1], the presence of circulatory insufficiency is implicated in this pathway.

To date, there are two cases of children in $\mathrm{HF}$ with description of CSR [5, 6]. Hoch and Barth reported a 6month-old boy with trisomy 21 and repaired atrioventricular septal defect who had pulmonary hypertension presented, had increased lethargy, and had CSR features on PSG. Rao and Arens posted a case report online [6] with clinical sleep fragments of a 16-year-old obese boy with DCM and congestive heart failure requiring inotropic support. This patient had PSG features of CSR. Our case supports these findings, confirming the presence of CSR in association with severely impaired cardiac function, and further confirms the complete resolution of the sleep disordered breathing following heart transplantation.

In children, cardiomyopathy is a rare but serious and life-threatening condition with an annual incidence of 1.1$1.2 / 100,000$. Dilated cardiomyopathy is the dominant phenotype, with two-thirds of all cases remaining idiopathic after diagnostic evaluation. The prognosis of DCM in children is guarded with increased morbidity and mortality, and currently $32-46 \%$ of patients with DCM will either receive a heart transplant or die within five years of diagnosis. Our patient had severe DCM with severely impaired cardiac 
function and he received an urgent heart transplant because of his deteriorating condition.

Several adult case reports and series have shown resolution and improvement of CSR in patients with HF after heart transplant [9], occurring as early as 9 weeks after a heart transplant [10]. However, a proportion of patients will have persistent CSR or convert to obstructive sleep apnea despite normalization of cardiac function, for unclear reasons $[9,10]$, but it has been suggested that this could be related to increased weight after heart transplant [11]. The resolution of CSR features in our patient supports the concept that the phenomenon is typically driven by circulatory insufficiency in children, as in adults.

In conclusion CSR can be present in children with advanced heart failure. We speculate that it may contribute to the symptom progression in these patients and may be a risk factor for death for that reason, although there are insufficient cases to determine this at present. Resolution of CSR after pediatric heart transplant would be expected assuming no other contributory factors are identified. Prospective evaluation of patients for the presence of sleep related disordered breathing appears justified, even in the pediatric age group, when advanced heart failure is identified.

\section{Abbreviations}

CSA: Central sleep apnea

CAI: Central apnea index

CSR: Cheyne-Stokes respiration

DCM: Dilated cardiomyopathy

HF: Heart failure

LVEF: Left ventricular ejection fraction

OSA: Obstructive sleep apnea

OAHI: Obstructive apnea-hypopnea index

PSG: Polysomnography

SDB: Sleep disordered breathing.

\section{Competing Interests}

The authors declare that they have no competing interests.

\section{Authors' Contributions}

All authors were involved in the writing and editing of the paper. All authors read and approved the final paper.

\section{References}

[1] D. Yumino and T. D. Bradley, "Central sleep apnea and CheyneStokes respiration," Proceedings of the American Thoracic Society, vol. 5, no. 2, pp. 226-236, 2008.

[2] R. B. Berry, R. Brooks, C. E. Gamaldo, S. M. Harding, C. L. Marcus, and B. V. Vaughn, The AASM Manual for the Scoring of Sleep and Associated Events: Rules, Terminology and Technical Specifications, Version 2.0.1, American Academy of Sleep Medicine, Darien, Ill, USA, 2013, http://www.aasmnet.org.

[3] P. A. Lanfranchi, A. Braghiroli, E. Bosimini et al., "Prognostic value of nocturnal Cheyne-Stokes respiration in chronic heart failure," Circulation, vol. 99, no. 11, pp. 1435-1440, 1999.
[4] T. Brack, I. Thüer, C. F. Clarenbach et al., "Daytime CheyneStokes respiration in ambulatory patients with severe congestive heart failure is associated with increased mortality," Chest, vol. 132, no. 5, pp. 1463-1471, 2007.

[5] B. Hoch and H. Barth, "Cheyne-stokes respiration as an additional risk factor for pulmonary hypertension in a boy with trisomy 21 and atrioventricular septal defect," Pediatric Pulmonology, vol. 31, no. 3, pp. 261-264, 2001.

[6] H. Rao and R. Arens, A 16-Year Old Obese Boy with Heart Failure and Sleep Apnea, 2012, https://www.thoracic.org/professionals/ clinical-resources/sleep/sleep-fragments/2012-may.php.

[7] S. Al-Saleh, P. F. Kantor, N. K. Chadha, Y. Tirado, A. L. James, and I. Narang, "Sleep-disordered breathing in children with cardiomyopathy," Annals of the American Thoracic Society, vol. 11, no. 5, pp. 770-776, 2014.

[8] S. Javaheri, "Sleep disorders in systolic heart failure: a prospective study of 100 male patients. The final report," International Journal of Cardiology, vol. 106, no. 1, pp. 21-28, 2006.

[9] D. R. Mansfield, P. Solin, T. Roebuck, P. Bergin, D. M. Kaye, and M. T. Naughton, "The effect of successful heart transplant treatment of heart failure on central sleep apnea," Chest, vol. 124, no. 5, pp. 1675-1681, 2003.

[10] S. A. Thalhofer, U. Kiwus, and P. Dorow, "Influence of orthotopic heart transplantation on breathing pattern disorders in patients with dilated cardiomyopathy," Sleep and Breathing, vol. 4, no. 3, pp. 121-125, 2000.

[11] S. Javaheri, W. T. Abraham, C. Brown, H. Nishiyama, R. Giesting, and L. E. Wagoner, "Prevalence of obstructive sleep apnoea and periodic limb movement in 45 subjects with heart transplantation," European Heart Journal, vol. 25, no. 3, pp. 260-266, 2004. 


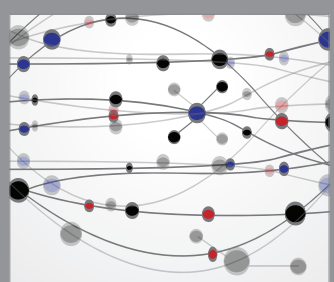

The Scientific World Journal
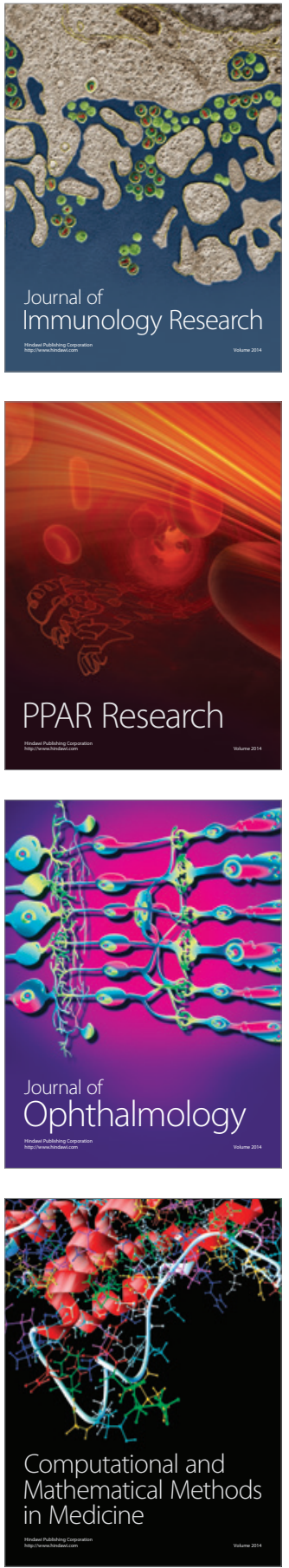

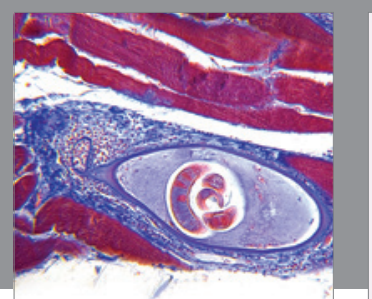

Gastroenterology Research and Practice

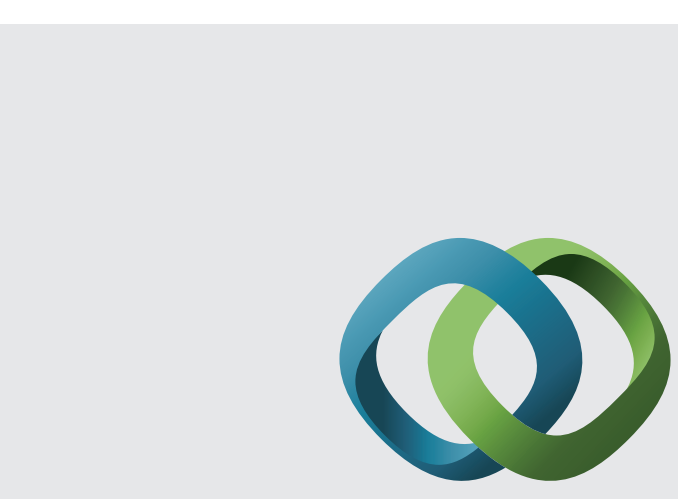

\section{Hindawi}

Submit your manuscripts at

http://www.hindawi.com
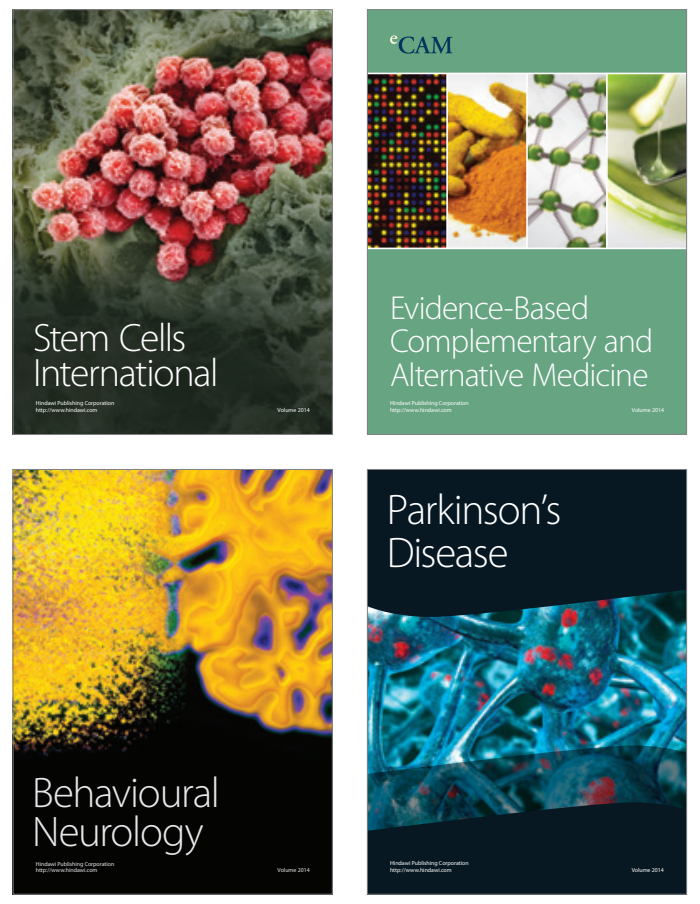
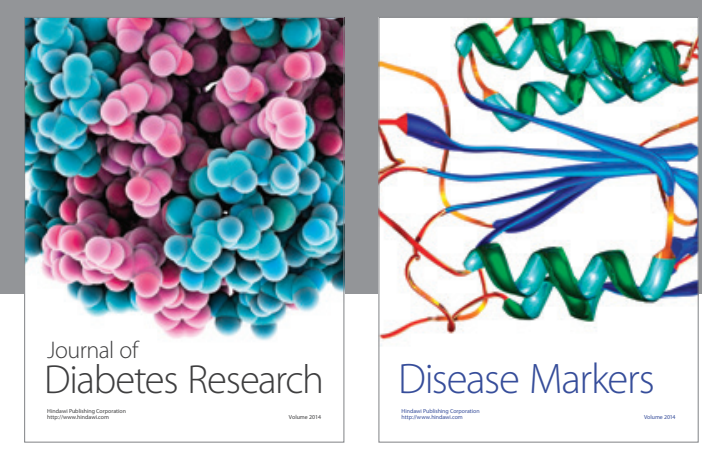

Disease Markers
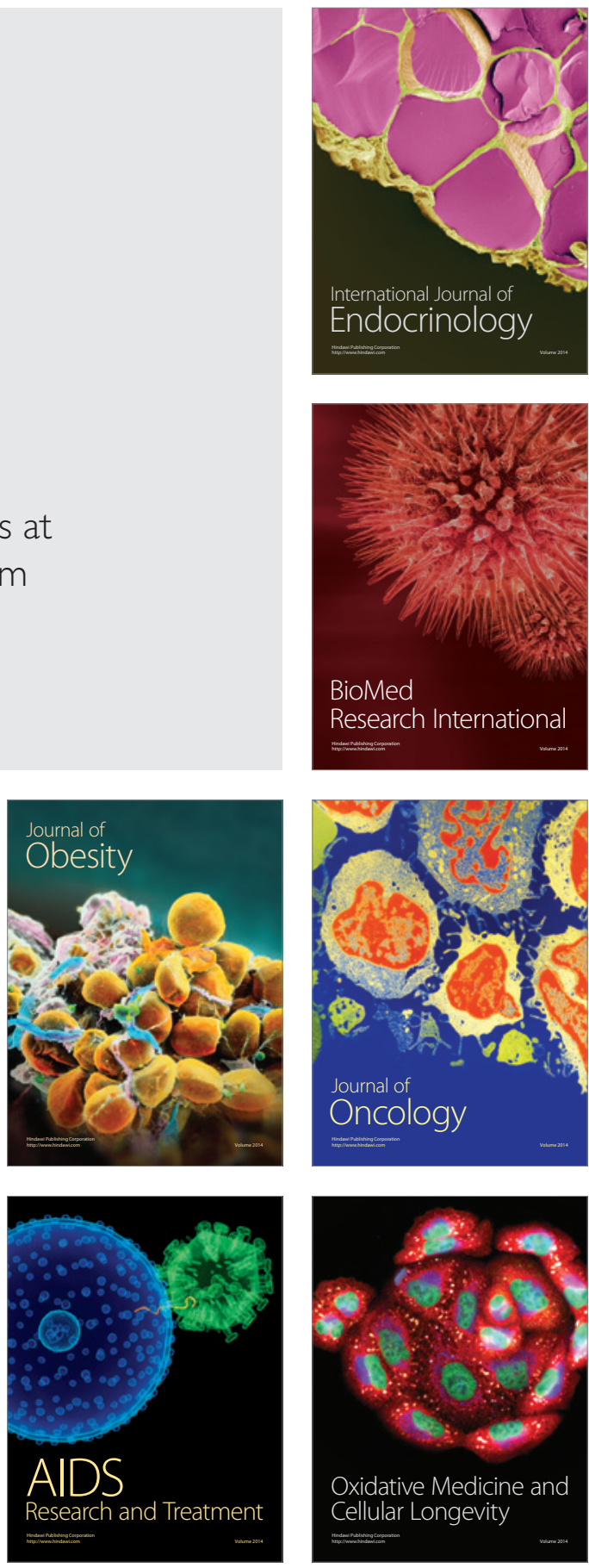